\title{
Current Testing of CMOS Combinational Circuits with Single Floating Gate Defects
}

\author{
VICTOR H. CHAMPAC ${ }^{\mathrm{a}, *}$ and JOAN FIGUERAS ${ }^{\mathrm{b}}$ \\ ${ }^{a}$ Instituto Nacional de Astrofísica, Optica y Electrónica, Grupo de Diseño de CI, Apdo. Postal 51 y 216, 72000 Puebla, Pue., Mexico; \\ ${ }^{\mathrm{b}}$ Universitat Politècnica de Catalunya, Departament d'Enginyeria Electrónica, Diagonal 649, Planta 9, 08028 Barcelona, Spain
}

\begin{abstract}
The behavior of basic CMOS combinational gates in the presence of a floating gate defect is characterized in order to investigate its detectability by $I_{D D Q}$. The defect is modeled at the circuit level by the poly-bulk and metal-poly capacitances, which determine the quiescent power supply current consumption $\left(I_{D D Q}\right)$ of the defective circuit. The testing implications on the type of defective gate are studied. Experimental measures have been made on basic CMOS combinational modules designed with intentional floating gate defects. A good agreement is observed between the simulation results and the experimental data. A conventional ATPG for stuck-at faults is used to obtain the required exciting vector to test the floating gate defects by $I_{D D Q}$ Testing.
\end{abstract}

Keywords: Defect modeling, floating gate defect, opens, current testing, exciting vectors, test pattern

\section{INTRODUCTION}

The class of defects due to opens causing a single transistor gate to float (Floating Gate Defect) [1] [2] [3] [4] [5] are physical failures that are not adequately represented by the stuck-at model. An open in the conductive path to the gate can be produced by contamination during manufacturing (spot defects) [6]. In addition, opens can also be produced by microcracks at the step oxide. In this work large breaks are studied so that tunneling effects (studied by Henderson et al. [7]) in the breaks can be considered negligible.

The floating gate defect has sometimes been modeled by the stuck-open model [8], assuming that the defective transistor does not conduct. However, re- cent research results [1] [2] [3] [4] [5] [9] show that the floating gate transistor may conduct in a wide range of defect topologies. It has been shown that the behavior of a floating gate transistor is influenced by several factors. The floating gate gets a voltage that depends on the coupling capacitances of the transistor device and the surrounding circuitry [1] [2] [3] [4] [5] [9]. In this work, the floating gate defect will be characterized at the circuit level taking into account the technology and topology of the circuit, and the physical location of the break. Our objective is to investigate the detectability of the floating gate defects by Current Testing [10] [11] [12]. Free-defective CMOS static circuits present a high impedance path between the power supply and ground in its quiescent state.

*Corresponding author. Fax: 52-22-470517. E-mail: champac@inaoep.mx 
Consequently, the quiescent power supply current consumption $\left(I_{D D Q}\right)$ is very low. A current path between $V_{D D}$ and $G_{N D}$ in the quiescent state, established by an input exciting vector, may exist if a floating gate transistor conducts when it should be "OFF" during a fault-free operation. The increase in the $I_{D D Q}$ can be used to detect the defect. The influences of the gate topology (i.e. inverter, Nand, Nor) on the $I_{D D Q}$ detectability is investigated. The most relevant aspects affecting the $I_{D D Q}$ are determined.

In the next section, the electrical model of the floating gate transistor with its coupling capacitances is presented. The parameters influencing the degree of conduction of the defective transistor are identified. Next, the behavior of a simple inverter chain with a floating gate defect is examined. The quiescent power supply current consumption is characterized in terms of the defect parameters. The analysis is extended to basic CMOS combinational modules to study the dependence of the gate structure on the $I_{D D Q}$ detectability. Basic CMOS combinational gates with intentional floating gate defects have been built and their $I_{D D Q}$ measured with the HP4145B Semiconductor Parameter Analyzer to compare with the predicted $I_{D D Q}$ levels using our modeling technique. A method to generate the exciting vectors to test the floating gate defects by Current Testing, using a standard ATPG for stuck-at faults, is proposed. Finally, the conclusions of the work are presented.

\section{FLOATING GATE DEFECT MODEL}

In this work, large opens in the transistor gate conducting path are considered. The poly path is broken and the electrical connectivity to the gate is lost (See Figure 1). It has been shown that the behavior of a floating gate transistor is rather complex because the floating node will get a voltage that depends on the coupling capacitances to the transistor gate and on the structure of the transistor itself [1] [2] [3] [4] [5] [9].

The voltage induced at the floating gate depends on several factors: a) The location of the break failure influences the values of two capacitances:

- The poly-bulk capacitance $C_{p b}$ (poly-well capacitance for the Pmos transistors) that depends on the length of the poly path that goes from the gate to the location of the break (See Figure 1) as well as on the gate-substrate overlap capacitance $\left(C_{g b o}\right)$. One terminal of these capacitances is connected to the bulk voltage (Nmos), or else to the n-well voltage (Pmos). For higher $C_{p b}\left(C_{p w}\right)$ the degree of conduction of an n-channel (p-channel) floating gate transistor decreases.

- In addition, the floating poly may be influenced by an overlapping metal track, which gives the metalpoly capacitance $\left(C_{m p}\right)$. This capacitance depends on the overlapped metal-poly (floating) areas. It is assumed that there exists a controllable signal $\left(V_{M}\right)$ in the metal track. For higher $C_{m p}$ and with $V_{M}=$ $5 \mathrm{~V}\left(V_{M}=0 \mathrm{~V}\right)$, the degree of conduction of an $\mathrm{n}$-channel (p-channel) floating gate transistor increases.

b) The drain, source overlap capacitances, $C_{g d o}$ (gate-drain overlap) and $C_{g s o}$ (gate-source overlap). The gate-drain (gate-source) overlap capacitance has one end to the drain (source) voltage $V_{D}$ $\left(V_{S}\right)$ and the other end has the gate voltage as indicated in Figure 2.

c) The influence of the transistor, modeled by the charge induced at the gate [5] [9]. It depends on the operation region of the defective transistor and on its structure.

In Figure 2, a circuit diagram of the coupling capacitances of a floating gate transistor is shown.

In the next section, the behavior of basic CMOS combinational modules in the presence of a single floating gate defect will be analyzed.

\section{CURRENT EXCITATION IN CMOS COMBINATIONAL MODULES}

The behavior of an inverter chain with a floating gate defect is analyzed. Special attention is paid to the 


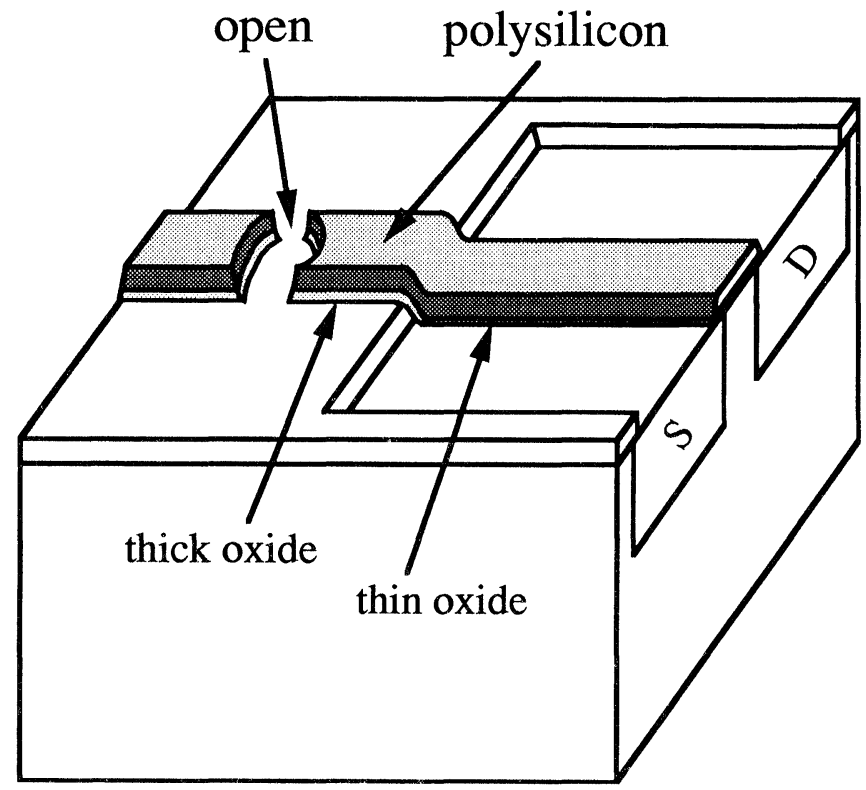

FIGURE 1 Transistor with an open at the poly track.

detectability of the floating gate defect by $I_{D D Q}$ Testing. Next, the testing implications in other type of gates are studied.

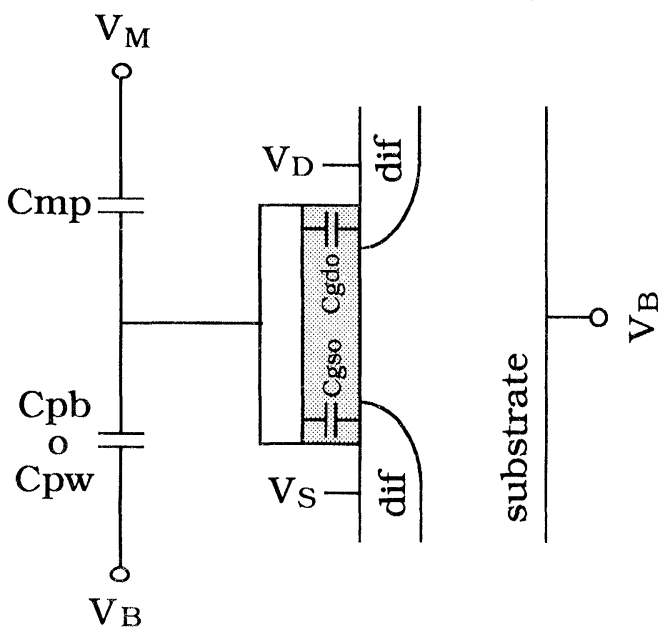

FIGURE 2 Floating gate transistor with the coupling capacitances.

\subsection{Current Testability of a Single Floating Gate Defect in an Inverter Chain}

A CMOS static inverter chain with an open path in a transistor gate is considered. The electrical model of the faulty circuit is indicated in Figure 3, where the defective transistor is located in the Nmos transistor of the first inverter and may be influenced by the voltage at the metal track through the capacitive coupling. Large opens are assumed so that the poly track cannot be influenced significantly by the input signal $\left(V_{I N}\right)$. First, the transfer capabilities $V_{O}$ versus $V_{I N}$ and $I_{D D}$ versus $V_{I N}$ of the defective inverter are examined (label $I_{D D}$ is used for the quiescent current varying with the power supply). Next, the quiescent power supply current consumption in terms of the defect parameters $\left(C_{p b}, C_{m p}\right)$ is characterized.

In an inverter with a floating gate defect in the Nmos transistor, when $V_{I N}$ is zero Volts there will exist a low impedance path from the power supply to ground if the floating gate gets enough voltage. This situation can be observed in Figure 4, where typical 


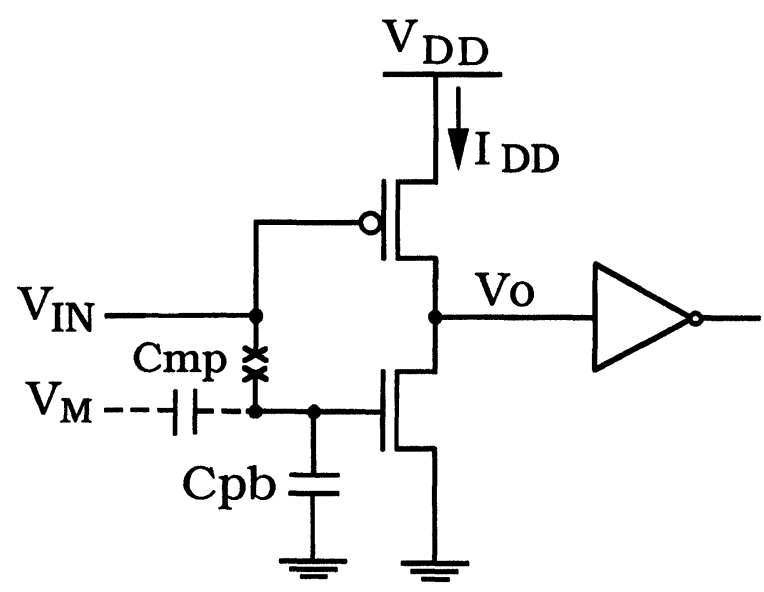

FIGURE 3 Inverter with a floating gate Nmos transistor.

curves for the output voltage $V_{O}$ of the defective inverter and the power supply current $\left(I_{D D}\right)$ are shown. For $V_{I N}=0 \mathrm{~V}$, the output voltage of the faulty inverter is lowered (See Figure 4) but it can be interpreted as a "good high logic level" by the next stage for a wide range of the defect parameters [3] [5] [13].

Taking into account that a fraction of the output voltage is induced at the floating gate, the output voltage and the power supply current $\left(I_{D D}\right)$ of the defective transistor decrease as $V_{I N}$ increases (See Figure 4). However, $V_{O}$ does not discharge completely to ground; it remains charged, if leakages are not taken into account, to a certain value. At that

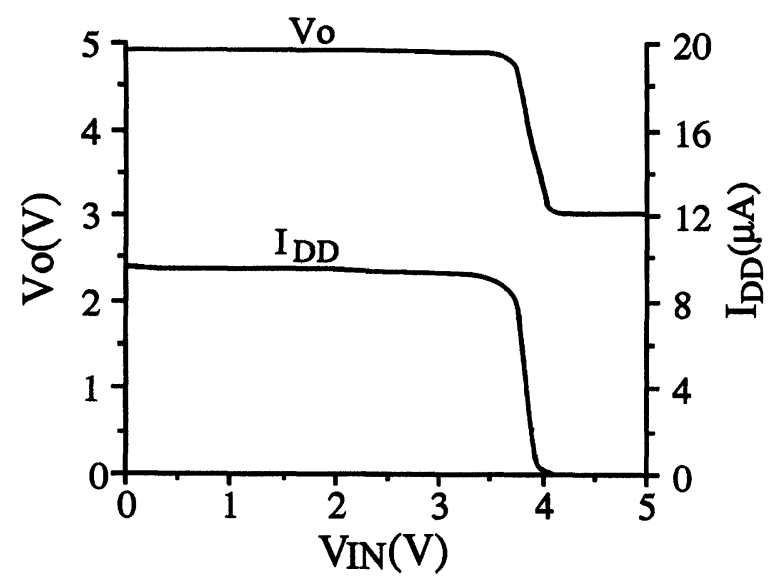

FIGURE 4 Typical Spice transfer curves for an inverter with a floating gate Nmos transistor. remanent $V_{O}$ value, it is induced the threshold voltage at the floating gate transistor [5]. The intermediate voltage may be interpreted as a high or a low logic level by the next stage depending on its threshold gate voltage. This depends on the relative sizes of the Pmos and Nmos transistors and on type of gate. Due to this, Logic Testing based on the stuck-open model may not detect some classes $\left(C_{p b}, C_{m p}\right)$ of floating gate defects [3] [5] [9]. When the floating poly is significantly influenced by a metal track $C_{m p}$, the voltage at the output of the defective inverter may be discharged very close to ground [5].

Now, the $I_{D D Q}$ detectability of the floating gate defect located in an Nmos transistor of an inverter chain is investigated. In this test methodology, the defect is excited by an input vector that creates a low impedance path from the power supply to ground if the floating gate gets enough voltage. For the inverter case, the input vector is $V_{I N}=0 \mathrm{~V}$. The quiescent power supply current consumption $I_{D D Q}$ is characterized in terms of the defect parameters $C_{p b}$ and $C_{m p}$. We consider two inverters with different sized transistors. One of them corresponds to a symmetric inverter of a minimum size allowed by the technology (ES2's Dual Layer Metal, $1.5 \mu \mathrm{m}$, n-Well CMOS) $W_{N}$ $=3 \mu \mathrm{m}$ and $W_{P}=10.8 \mu \mathrm{m}$. The other corresponds to an inverter from the ES2's library (same technology as before) $W_{N}=W_{P}=15.2 \mu \mathrm{m}$.

The values of the capacitances $C_{p b}$ and $C_{m p}$ determine the degree of conduction of the floating gate transistor. Depending on $C_{p b}$ and $C_{m p}$ values, the quiescent power supply current through the inverter can be estimated. For the standard cell inverter, significant $I_{D D Q}$ values exist in the entire range $\left(0 \leq C_{p b} \leq\right.$ $5 \mathrm{fF}, 0 \leq C_{m p} \leq 3 \mathrm{fF}$ ) of the defect parameters (See Figures 3 and 5a). It must be noted that even for the worst case defect parameters $\left(C_{m p}=0\right.$ and $C_{p b}$ large), high values of $I_{D D Q}$ are found. Hence, the floating gate defects located in this inverter are detectable by $I_{D D Q}$ Testing.

For the minimum sized inverter, a large class of the floating defects may be detected by Current Testing (See Figure 5b). The $I_{D D Q}$ depends on the defect parameters. For large values of $C_{p b}$, the floating gate transistor may operate in the subthreshold region or 


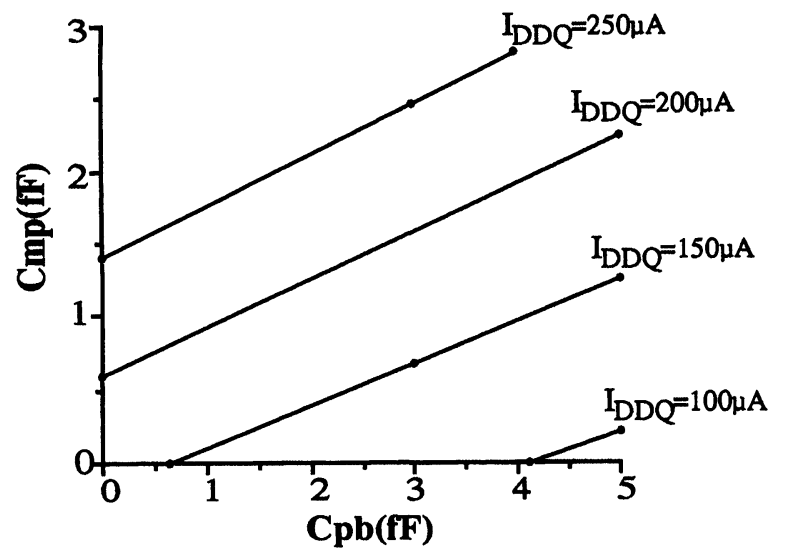

a) Standard cell library inventor

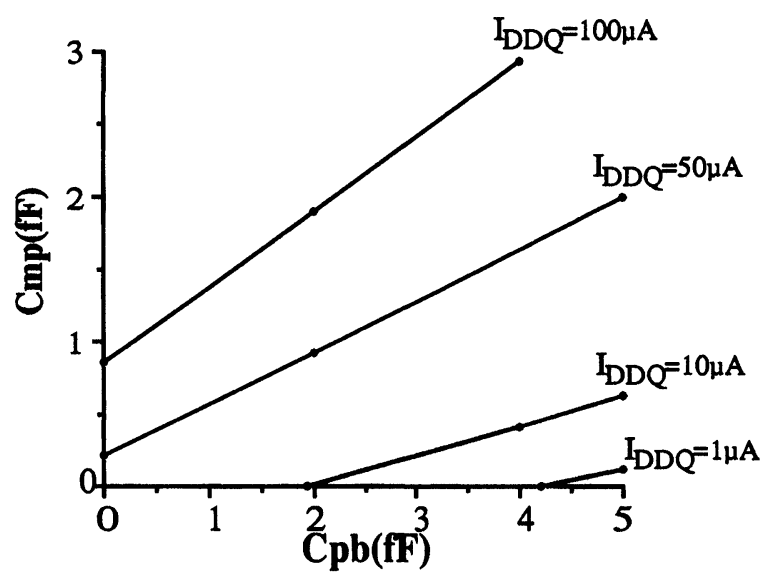

b) Minimum sized inverter

FIGURE $5 I_{D D Q}$ characterization on the $C_{p b}-C_{m p}$ plane, $V_{I N}=$ $0 \mathrm{~V}, V_{M}=5 \mathrm{~V}$.

may conduct very weakly, as can be observed in Figure $5 \mathrm{~b}$. These defects may not be detected by $I_{D D Q}$ Testing if the sensor is not able to discriminate low levels of $I_{D D Q}$.

Next, we consider the detectability of the floating gate defect in other types of basic CMOS gates.

\subsection{Current Testability of Floating Gate Defects in Basic CMOS Gates}

In this subsection, the $I_{D D Q}$ dependence on the position of the floating gate transistor in Nand and Nor modules is analyzed. It is assumed that the required exciting vector is applied.
In an inverter with a floating gate defect in the Nmos transistor, the source voltage of the defective transistor is at ground potential and the drain is the gate output. However, this is not true in multiple input gates. In a Nand gate, a current path from the power supply to ground is created via series connected Nmos transistors with one Pmos transistor. Due to this, the voltages at the drain and source of the defective transistor, depend on the gate inputs and on the position of the floating gate transistor. As shown above, the voltages at the floating gate transistor determine the conductivity of the defective transistor and the $I_{D D Q}$ value of the gate.

In a three-input Nand gate there are three possible positions for a floating gate Nmos transistor: upper $(f 1)$, lower (f3) and middle (f2) transistors (See Figure 6). An inverter with similar sized transistors as the Nand gate has been taken as reference to compare the qualitative behavior of the different cases.

The possible defective Nmos cases for the threeinput Nand gate are:

1. Defect in the upper transistor $(f 1)$.- The conductance of the defective upper transistor of the Nand gate is lower than for a similar defect (same $C_{p b}$, $C_{m p}$ ) located in an inverter due to the non zero value of its source voltage. This decreases the de-

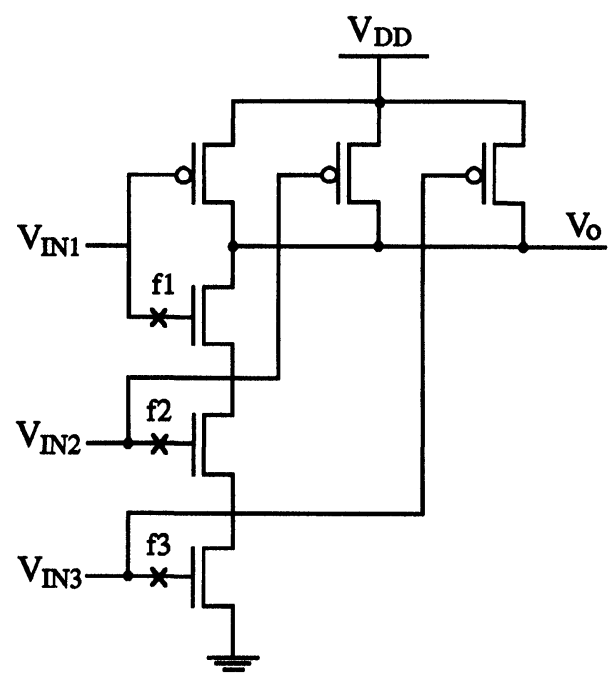

FIGURE 6 Three input nand gate with the possible locations of the floating gate Nmos transistor. 
gree of conduction of the defective upper transistor owing to the body effect. In addition, the voltages $\left(V_{F G}-V_{S}\right)$ and $\left(V_{O}-V_{S}\right)$ are lower for greater voltages of the source voltage $V_{S}$. The value of the source voltage depends on the number of series connected transistors from the source of the defective transistor to ground. Due to the non zero value of the source voltage of the defective transistor, the $I_{D D Q}$ values in a defective Nand gate (defect $f 1$ ) are lower than those found for a similar defect in an inverter with the same sized transistors as the Nand gate (See Figure 7). The $I_{D D Q}$ decreases as there exists a greater number of series Nmos connected transistors in the current path.

2. Defect in the lower transistor (f3).- Owing to the fact that the drain of a transistor below the one that controls the output of the gate may get a maximum drain voltage of $V_{D D}-V_{T}$, the conductance of the lower Nmos transistor decreases significatively. In addition, the $I_{D D Q}$ decreases (See Figure 7) due to the drop voltages in the series connected transistors in the current path.

3. Defect in the middle transistor $(f 2)$.- The $I_{D D Q}$ for a defect located in the middle transistor is affected by a combination of the effects on the upper and the lower defective transistors. However, the increase in the voltage at its source is lower than for a defective upper transistor (See Figure 7).

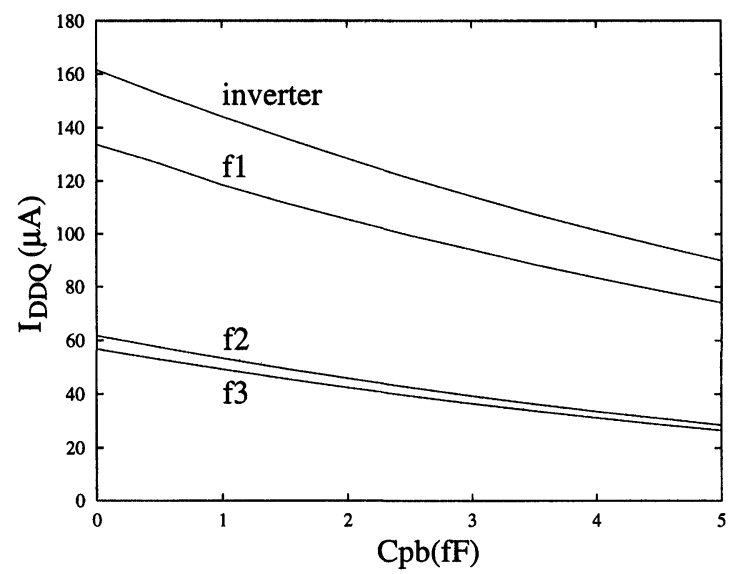

FIGURE $7 I_{D D Q}$ dependency with the position of the floating gate transistor in the Nand gate $W_{N}=W_{P}=15.2 \mu \mathrm{m}$ for all gates.
Now, let us consider a Nor gate. The defects located in a Nmos transistor of a Nor gate are affected by the drop voltages in the Pmos series connected transistors. Taking into account that the conductances of the designed Pmos transistors are in general lower than for the Nmos transistors, the $I_{D D Q}$ values are lower than those found in an inverter with the same sized transistors.

As it has been shown, the $I_{D D Q}$ has a significant dependence on the gate type and on the position of the defective transistor. In a Nand gate, for defects in an Nmos transistor with a relative large channel width, the $I_{D D Q}$ is lowered (See Figure 7) but the defects are still detectable by $I_{D D Q}$ Testing. However, for the defects in a Nmos transistor with a small size, the $I_{D D Q}$ decrease will affect the defect detectability significantly. This is analyzed comparing the $I_{D D Q}$ testability in the $C_{p b}-C_{m p}$ plane for an inverter with the Nmos defective transistor (See Figure 8a) and a three-input Nand gate with the defective lower Nmos transistor (See Figure 8b). The Nmos and Pmos transistors of the inverter and Nand gates have the same channel width $\left(W_{N}=3 \mu \mathrm{m}\right.$ and $\left.W_{P}=10.8 \mu \mathrm{m}\right)$. We assume a minimum quiescent current $\left(I_{D D Q}^{\text {mindet }}\right)$ of $1 \mu \mathrm{A}$ for $I_{D D Q}$ defect detection. In an inverter some defect characterizations considered detectable are not detectable in a Nand gate. Hence, the region in the $C_{p b^{-}}$ $C_{m p}$ plane that is $I_{D D Q}$ detectable in the inverter gate is larger than for a Nand gate (See Figures 8a and $8 b)$.

In summary, the defects located in Nmos transistors of small size below one that controls the output of the gate may have non significant $I_{D D Q}$ values. Hence, some classes of these defects may not be detected by $I_{D D Q}$ Testing.

In the next section, experimental measurements carried out on intentionally designed defective gates are presented.

\section{EXPERIMENTAL MEASUREMENTS}

Basic CMOS combinational gates with intentionally open gate defects have been designed and fabricated. 


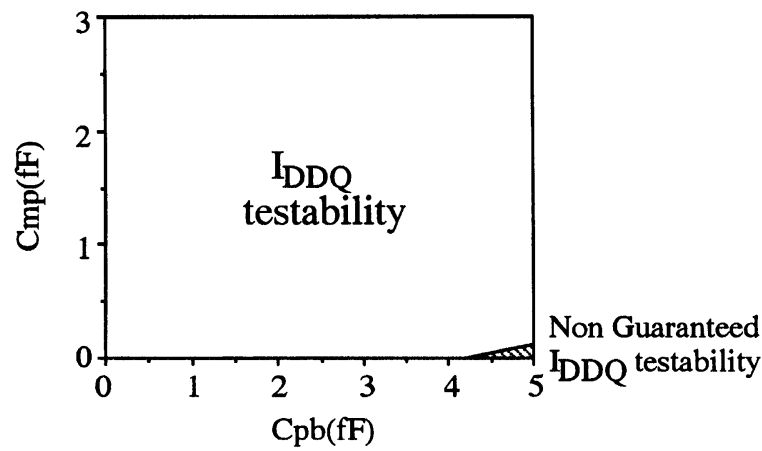

a) Defect in the Nmos of an inverter

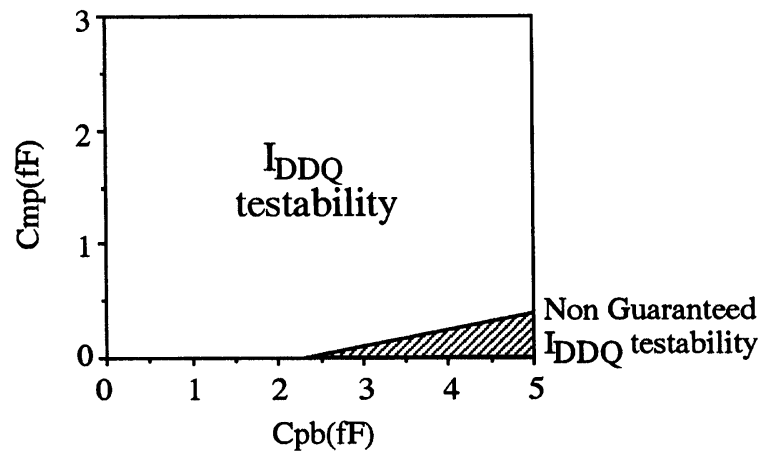

b) Defect in the lower Nmos of a three imput Nand

FIGURE $8 \quad I_{D D Q}$ testability in the $C_{p b}$ - $C_{m p}$ plane of an Nmos floating gate transistor of an inverter and a three-input Nand gate with a defect in the lower transistor, $W_{N}=3 \mu \mathrm{m}$ and $W_{P}=10.8 \mu \mathrm{m}$ $I_{D D Q}^{\text {mindet }}=1 \mu A$.

The polysilicon that normally controls the gate of a transistor is opened in the layout of the circuit. The open is designed in different places to study the effect of the poly-bulk capacitance $\left(C_{p b}\right)$ in the behavior of the circuit. The poly-floating track is crossed by a metal track to study the effect of the metal-poly capacitance $\left(C_{m p}\right)$. A photograph taken with an optical microscope of a Nand gate with a defect in the upper Nmos transistor and without influence $\left(C_{m p}=0\right)$ is shown in Figure 9. An HP4145B Semiconductor Parameter Analyzer has been used for the experimentation.

The defective circuits have been fabricated in ES2's Dual Layer Metal, 1.5 $\mu \mathrm{m}$, n-Well CMOS technology. All the transistors (Pmos and Nmos) have been designed with the same width $W_{N}=W_{P}=15.2 \mu \mathrm{m}$ and length of channel $L=1.6 \mu \mathrm{m}$.

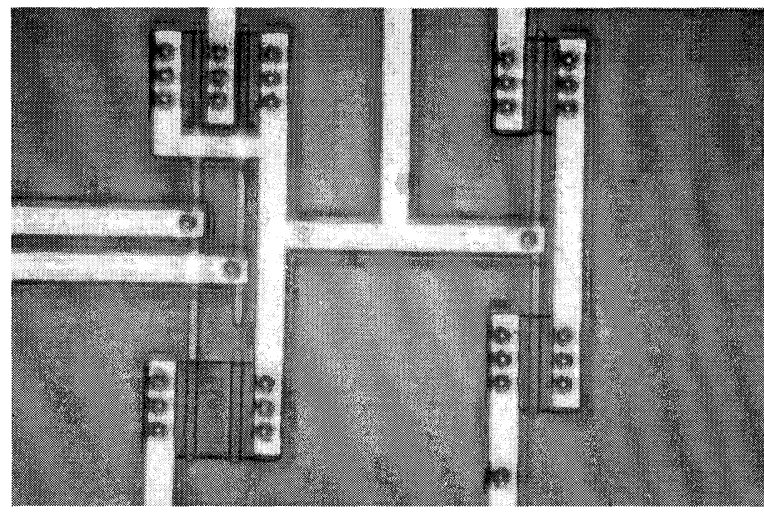

FIGURE 9 Photograph of a Nand gate with a floating gate defect in the upper Nmos transistor.

Our objective is to verify the proposed characterization with experimental measurements. The following aspects are considered:

1. The dependence of the quiescent current consumption on the parameters characterizing the defect $\left(C_{p b}, C_{m p}\right)$ [14]. This behavior is measured in defective inverters.

2. The dependence of the quiescent current consumption on the position of the the defective floating gate transistor. This effect is shown with measurements of inverters and of Nand gates designed with the defective transistor in different positions.

In Figure 10, the circuit used for the measurements

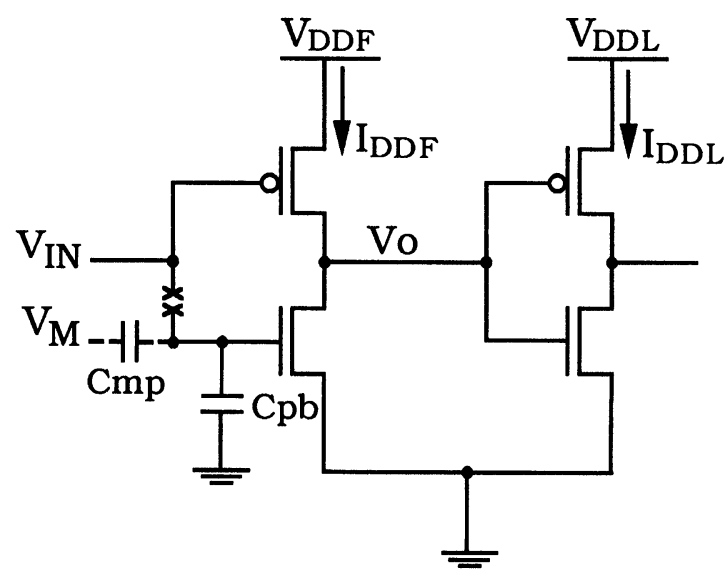

FIGURE 10 Circuit used for the measurement of the quiescent current at the defective inverter. 


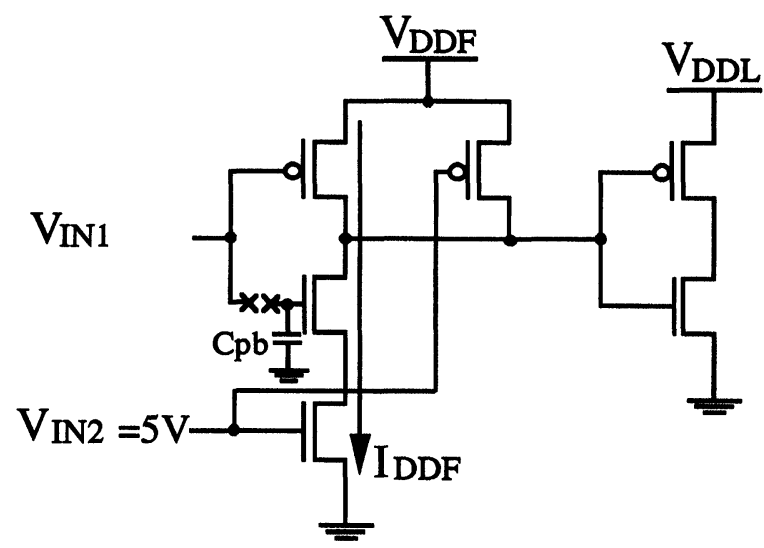

FIGURE 11 Circuit used for the measurement of the quiescent current at the defective Nand gate (defect in the upper Nmos transistor).

of the two inverter chain is shown. We have designed separately the $\mathrm{n}$-Well for each inverter, in order to be able to measure the quiescent current consumptions in the defective inverter $\left(I_{D D F}\right)$ separately of the quiescent current in the load inverter $\left(I_{D D L}\right)$. The set-up measurement for a defect located in the upper Nmos transistor of a Nand gate is shown in Figure 11.

- In Figure 12, the quiescent current consumption at the defective inverter $\left(I_{D D F}\right)$ versus the input voltage $\left(V_{I N}\right)$ is shown. This has been made for two non influenced cases $\left(C_{m p}=0\right)$ and one with influence. Significant values $I_{D D F}$ exists for $V_{I N}=$ $0 \mathrm{~V}$ exciting vector as can be observed in the Figure

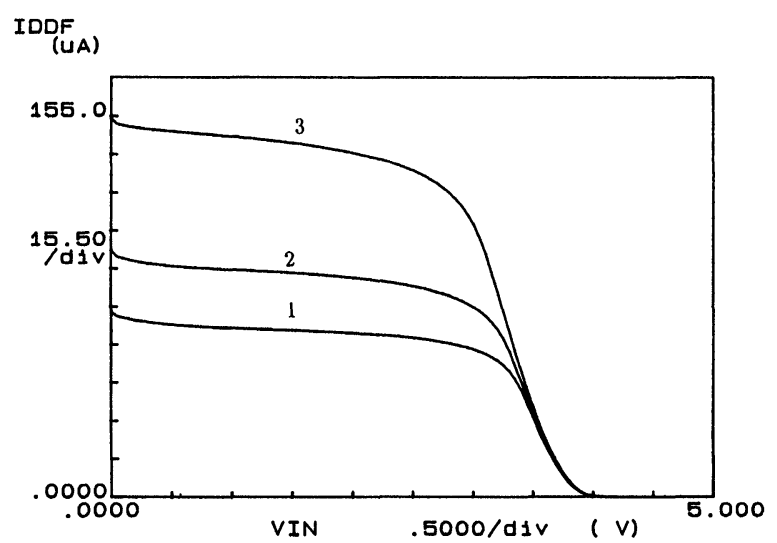

FIGURE 12 Quiescent current consumption through the defective inverter $I_{D D F}, 1: C_{p b}=3 \mathrm{fF}, 2: C_{p b}=1 \mathrm{fF}, 3: C_{p b}=3 \mathrm{fF} C_{m p}=$ $1 \mathrm{fF} V_{M}=5 \mathrm{~V}$.

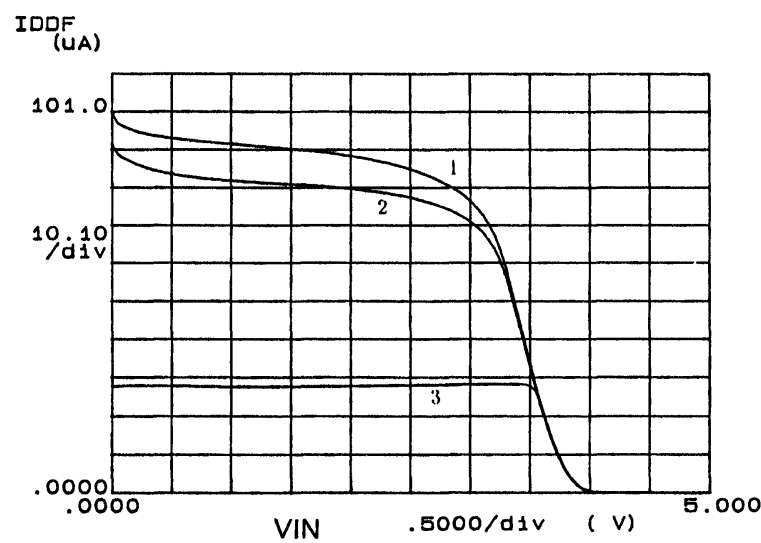

FIGURE 13 Quiescent current consumption for different positions of the floating gate transistor and the same $C_{p b}=1 \mathrm{fF}, 1$ : Inverter, 2: defect in the upper Nmos of the Nand, 3: defect in the lower Nmos of the Nand.

12. A significant dependence of the quiescent current on the defect parameters appears to exist. For higher $C_{p b}$ values, the $I_{D D F}$ decreases and when a significative influence exists the $I_{D D F}$ increases.

- Next, the dependence of the $I_{D D F}$ on the position of the defective transistor is studied. Two-input Nand gates have been designed with the defect located in the upper and lower Nmos transistors. In Figure 13, the quiescent current consumption in defective gates $\left(I_{D D F}\right)$ for the same defect characterization $C_{p b}=1 \mathrm{fF}$ is shown. In Figure 14, this is shown for $C_{p b}=3 \mathrm{fF}$. We have considered (See Figures 13

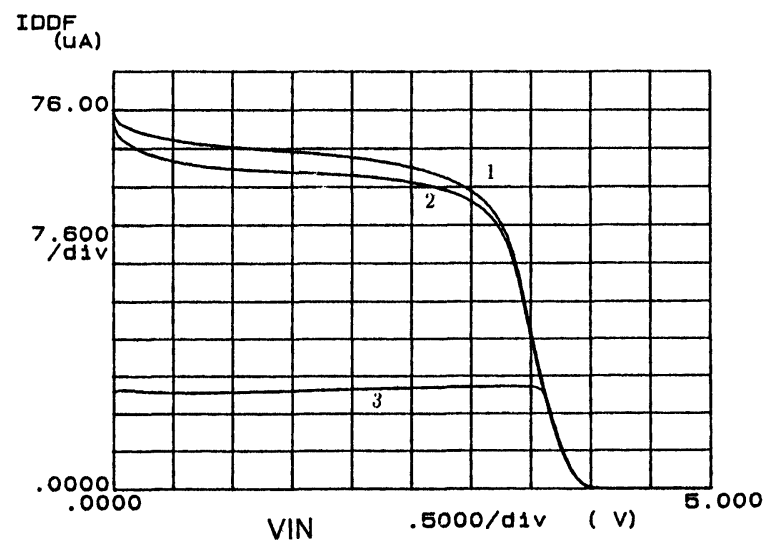

FIGURE 14 Quiescent current consumption for different positions of the floating gate transistor and the same $C_{p b}=3 \mathrm{fF}, 1$ : inverter, 2: defect in the upper Nmos of the Nand, 3: defect in the lower Nmos of the Nand. 
and 14) defects located in the Nmos transistor of an inverter (1), in the upper Nmos of a Nand gate (2) and in the lower Nmos of the Nand gate (3). It may be observed that the $I_{D D F}$ for a defect in the upper Nmos of the Nand gate is lower than that of a similar defect located in the inverter. When the defect is located in the lower Nmos transistor of the Nand gate, the quiescent current consumption decreases significantly as a consequence of the decrease in voltage at the drain of the defective transistor.

It must be noted that the channel width of the transistors is relatively large. The $I_{D D Q}$ values would be smaller for transistors of smaller sizes.

In the next section, we propose a method to generate the exciting vectors to test a floating gate transistor.

\section{LOGIC EXCITATION OF SINGLE FLOATING GATE DEFECTS}

In this section, a method to generate the exciting vectors to test the floating gate defects by $I_{D D Q}$ Testing, using an ATPG based in the stuck-at model, is proposed. This method is made for the non-influenced case.

The following conditions assure maximum $I_{D D Q}$ quiescent current: a) The drain voltage of the floating gate Nmos (Pmos) transistor must be maximized (minimized), this gives a greater (lower) induced voltage at the floating gate, by connecting it through a low impedance path to $V_{D D}\left(G_{N D}\right)$; b) The source voltage of the floating gate Nmos (Pmos) transistor must be minimized (maximized) by connecting it to $G_{N D}\left(V_{D D}\right)$.

To test a defect located in the Nmos (Pmos) transistor on an inverter (See Figure 15a) the conditions stated previously are satisfied by: a) The drain voltage is driven to its maximum (minimum) voltage making $V_{A}=0 \mathrm{~V}\left(V_{A}=5 \mathrm{~V}\right)$ b) The path from the source of the defective transistor to ground has to be completed. In this single case, this condition has been already satisfied by $V_{A}=0 \mathrm{~V}\left(V_{A}=5 \mathrm{~V}\right)$. At the log- ical level, these excitation conditions are equivalent to the detectability of the input $A$ stuck-at 1 (stuck-at 0 ) (See Figure 15b). The pattern generation just require the controllability at the input of the defective inverter with its output as a pseudo-primary output (Note that this just affect the netlist of the circuit).

For defects located in a Nand gate, the required vectors are the same set that detects the stuck-at 1 and stuck-at 0 faults in every input of the gate. The output of the defective gate is assumed to be made observable (pseudo-primary output). It can be shown that a set of $k+1$ vectors are sufficient to detect these stuck-at faults. Hence, $k+1$ vectors are sufficient to

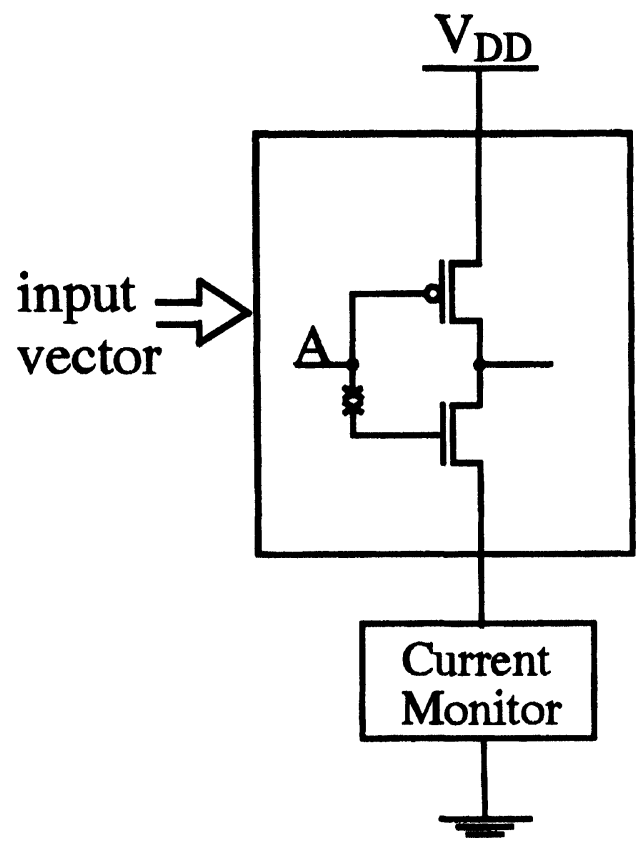

a) Current Testing

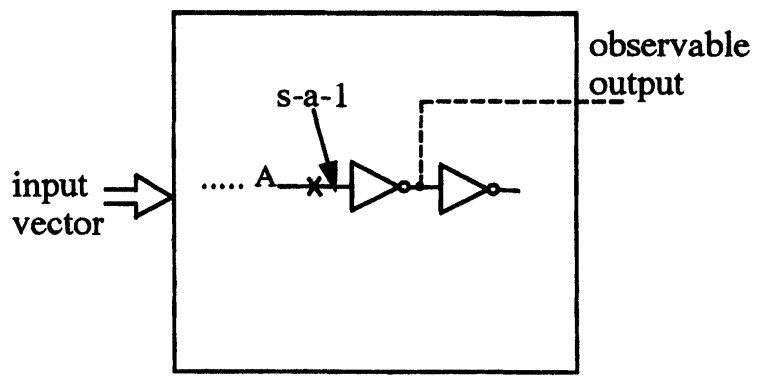

b) ATPG circuit

FIGURE 15 Test pattern generation to test a floating gate transistor located in an inverter by $I_{D D Q}$ testing. 
TABLE I Coverage for Floating Gate Defects (FGs).

\begin{tabular}{|c|c|c|c|c|c|c|}
\hline Circuit & FG & \# vectors & $\mathrm{FG}_{\mathrm{D}}$ & $\mathrm{FG}_{\mathrm{L}}$ & $\mathrm{C}_{\mathrm{D}}$ & $\mathrm{C}_{\mathrm{L}}$ \\
\hline c880 & 1802 & 21 & 1802 & 1802 & $100 \%$ & $100 \%$ \\
\hline c1355 & 2308 & 91 & 2308 & 2308 & $100 \%$ & $100 \%$ \\
\hline c1908 & 3446 & 114 & 3444 & 3444 & $99.94 \%$ & $100 \%$ \\
\hline c2670 & 5364 & 24 & 5314 & 5314 & $99.07 \%$ & $100 \%$ \\
\hline c3540 & 7504 & 54 & 7449 & 7460 & $99.27 \%$ & $99.85 \%$ \\
\hline c5315 & 11262 & 33 & 11258 & 11258 & $99.96 \%$ & $100 \%$ \\
\hline c6288 & 10112 & 20 & 10060 & 10060 & $99.49 \%$ & $100 \%$ \\
\hline c7552 & 15396 & 48 & 15370 & 15370 & $99.83 \%$ & $100 \%$ \\
\hline s208 & 374 & 30 & 374 & 374 & $100 \%$ & $100 \%$ \\
\hline s298 & 582 & 23 & 582 & 582 & $100 \%$ & $100 \%$ \\
\hline s344 & 644 & 14 & 644 & 644 & $100 \%$ & $100 \%$ \\
\hline s349 & 654 & 13 & 649 & 649 & $99.24 \%$ & $100 \%$ \\
\hline s382 & 682 & 24 & 682 & 682 & $100 \%$ & $100 \%$ \\
\hline s1196 & 2456 & 49 & 2456 & 2456 & $100 \%$ & $100 \%$ \\
\hline s1494 & 3902 & 53 & 3902 & 3902 & $100 \%$ & $100 \%$ \\
\hline s5378 & 8902 & 68 & 8857 & 8857 & $99.49 \%$ & $100 \%$ \\
\hline s9234 & 18714 & 95 & 18645 & 18645 & $99.63 \%$ & $100 \%$ \\
\hline
\end{tabular}

FG: Number of FGs.

$\mathrm{FG}_{\mathrm{D}}$ : Number of detected FGs.

$\mathrm{FG}_{\mathrm{L}}$ : Number of detectable FGs.

$\mathrm{C}_{\mathrm{D}}$ : Coverage considering all possible FGs. $\mathrm{C}_{\mathrm{D}}=\mathrm{FG}_{\mathrm{D}} / \mathrm{FG}$.

$\mathrm{C}_{\mathrm{L}}$ : Coverage considering all detectable FGs. $\mathrm{C}_{\mathrm{L}}=\mathrm{FG}_{\mathrm{D}} / \mathrm{FG}_{\mathrm{L}}$.

detect the single floating gate defects of the Nand gate by $I_{D D Q}$ Testing. Similarly, as far as Nor gates are concerned, $k+1$ vectors are also sufficient to test the possible defective transistors using $I_{D D Q}$ Testing.

The previous test pattern generation method has been applied to the combinational ISCAS benchmarks circuits [15] and to the combinational sections of the sequential ISCAS benchmarks circuits [16]. Using an automatic procedure the AND and OR gate are replaced by equivalent Nand-Not and Nor-Not configurations respectively. Table I shows the coverage for floating gate defects without influence and Table II the executions time using CUTEGENS [17] on a SUN SPARC station 2.

In Table I, $F G_{\mathrm{D}}$ is the number of the floating gate transistors for which are satisfied the test vector excitations conditions stated before. There exist 11 floating gates faults (c3540) for which it has not been demonstrated that there is not vector satisfying the excitation conditions. A pessimistic evaluation has been assumed and these floating faults are considered as possible detectables faults $\left(F G_{\mathrm{L}}\right)$. High coverages of the floating gate transistors are obtained as can be observed in the last two column of the Table I.

\section{CONCLUSIONS}

It has been shown that a transistor with a floating gate defect (large opens) conducts for a large class of realistic situations. The capacitive coupling may induce enough voltage at the floating gate to force the operation of the defective transistor above the threshold creating a channel. The location of the poly break, modeled by the poly-bulk $\left(C_{p b}\right)$ and metal-poly $\left(C_{m p}\right)$

TABLE II Execution times for CUTEGENS [17] on a SUN SPARC station 2.

\begin{tabular}{lccccccccc}
\hline Circuit & $\mathrm{c} 880$ & $\mathrm{c} 1355$ & $\mathrm{c} 1908$ & $\mathrm{c} 2670$ & $\mathrm{c} 3540$ & $\mathrm{c5315}$ & $\mathrm{c6288}$ & $\mathrm{c} 7552$ \\
Time $(\mathrm{s})$ & 26.5 & 132.7 & 230.3 & 128.5 & 439.2 & 337.7 & 1129.0 & 449.0 & $\mathrm{~s}$ \\
\hline Circuit & $\mathrm{s} 208$ & $\mathrm{~s} 298$ & $\mathrm{~s} 344$ & $\mathrm{~s} 349$ & $\mathrm{~s} 382$ & $\mathrm{~s} 1196$ & $\mathrm{~s} 1494$ & $\mathrm{~s} 5378$ \\
Time (s) & 17.6 & 17.1 & 15.3 & 16.0 & 22.7 & 66.7 & 77.5 & 358.4 & 1271.1 \\
\hline
\end{tabular}


capacitances determines the $I_{D D Q}$ of the defective gate. In addition, the $I_{D D Q}$ depends on the position of the floating gate transistor in the defective gate considered. It has been shown that defective floating gate transistors of small size, located below the one controlling the output of the gate give lower levels of $I_{D D Q}$. Experimental data on defective circuits with different locations of the breaks have been obtained. The measured quiescent current depends on the break location and on the position of the defective floating gate transistor in each type of gate studied. The measurements show good agreement with the predicted $I_{D D Q}$ levels for the circuits analyzed. The required excitation conditions of the floating gate defects has been translated to the stuck-at controllability at the inputs of the gate. This allows the generation of the excitation vectors to test the floating gate defects by $I_{D D Q}$ Testing using a conventional ATPG. In general, gates (inverter, Nand, Nor) with $k$ inputs need $k+1$ vectors to test the possible floating gate transistor.

\section{Acknowledgements}

The authors thank Eugenio Isern for providing data fault simulation using CUTEGENS. This work has been partially supported by the Project ARCHIMEDES (ESPRIT III Basic Research Project No 7107) and by the Comisión Interministerial para la Ciencia y Tecnología(CICYT) No TIC 94-0561.

\section{References}

[1] Renovell, M. and Cambon, G. (1986). "Topology Dependence of Floating Gate Faults in MOS Circuits", Electronics Letters, Vol.22, No 3, (January), pp. 152-153.

[2] Maly, W., Nag, P. K. and Nigh, P. (1988). "Testing Oriented Analysis of CMOS ICs with Opens", Proc. Int. Conf. on Computer-Aided Design, pp. 344-347.

[3] Champac, V. H., Rodrígues-Montañés, R., Segura, J. A., Figueras, J. and Rubio, J. A. (1991). "Fault Modelling of Gate Oxide Short, Floating Gate and Bridging Failures in CMOS Circuits", European Test Conference, pp. 143-148.

[4] Renovell, M. and Cambon, G. (1992). "Electrical Analysis and Modeling of Floating-Gate Fault", IEEE Transactions on Computer-Aided Design, Vol. 11, No. 11, (November), pp. $1450-1458$.

[5] Victor H. Champac, (1993). "Caracterización del defecto de puerta flotante y su detección en circuitos CMOS digitales",
Ph.D. dissertation, Universitat Politècnica de Catalunya, Spain, March.

[6] Maly, W. (1985). "Modelling of Lithography Related Yield Losses for CAD of VLSI Circuits", IEEE Transactions on Computer-Aided Design, Vol. CAD-4, No. 3, (July), pp. 166-177.

[7] Henderson, C. L., Soden, J. M. and Hawkins, C. F. (1991). "The behavior and Testing Implications of CMOS IC Logic Gate Open Circuits", International Test Conference, pp. 302-310.

[8] Wadsack, R. L. (1978). "Fault Modeling and Logic Simulation of CMOS and MOS Integrated Circuits", The Bell System Technical Journal, vol.57, (May-June), pp. 1449-1474

[9] Champac, Victor H., Rubio, J. A. and Figueras, J. (1994). "Electrical Model of the Floating Gate Defect in CMOS ICs: Implications on $I_{D D Q}$ Testing", IEEE Transactions on Computer-Aided Design, Vol. 13, (March), pp. 359-369.

[10] Maly, W. and Nigh, P. (1988). "Built-In Current Testing: Feasibility Study", Proc. Int. Conf. on Computer-Aided Design, pp. 340-343.

[11] Hawkins, C. F., Soden, J. M., Fritzemeier R. R. and Horning, L. K. (1989). "Quiescent Power Supply Current Measurement for CMOS IC Detection", IEEE Transactions on Industrial Electronics, Vol. 36, No. 2, (May), pp. 211-218

[12] Soden, J. M., Hawkins, C. F., Gulati R. K. and Mao, W. (1992). "I $I_{D D O}$ Testing: A Review", J. Electronic Testing: Theory and Applications (Jetta), Vol. 3, No. 4, (December), pp. 291-303.

[13] Rodríguez-Montañés, R., Segura, J. A., Champac, V. H., Figueras, J. and Rubio, J. A. (1991). "Current vs Logic Testing of Gate Oxide Short, Floating Gate and Bridging Failures in CMOS", International Test Conference, pp. 510519.

[14] Segura, J. A., ' Champac, V. H., Rodríguez-Montañés, Figueras, J. and Rubio, J. A. (1992). "Quiescent Current Analysis and Experimentation of Defective CMOS Circuits", J. Electronic Testing: Theory and Applications (Jetta), Vol. 3, No. 4, (December), pp. 337-348.

[15] Brglez F. and Fujiwara, H. (1985). "A neutral netlist of 10 combinational benchmark circuits and a target translator in fortran", IEEE Int. Symp. on Circuits and Systems, pp. 663 698.

[16] Brglez, F., Bryan D. and Kozminski, K. (1989). "Combinational Profiles of Sequential Benchmark Circuits", IEEE Int'l Symp. on Circuits and Systems, pp. 1929-1934.

[17] Isern, Eugenio and Figueras, Joan, (1993). "Test Generation with High Coverages for Quiescent Current Test of Bridging Faults in Combinational Circuits", International Test Conference, $\mathrm{pp}$ 73-82.

\section{Authors' Biographies}

Victor H. Champac was born in Perú in 1964. He received the Electronic Engineering degree from the Universidad Autónoma de Nuevo León (México) and the Ph.D. degree (Electronic Technology) from the Universitat Politècnica de Catalunya (UPC), Spain. From 1988 to 1993, he was associate professor of the Electronic Engineering Department at the UPC. He is 
currently titular professor at the Instituto Nacional de Astrofísica, Optica y Electrónica-INAOE (Mexico). His research interests include VLSI design and test, defect and fault models and testing mixed signal circuits.

Joan Figueras received his Industrial Engineering (Electrical) degree from the Universitat Politècnica de Catalunya and the Ms.C. (Electrical) and Ph.D. (Computer Information and Control) degrees from the University of Michigan were he was a Fullbright Scholar and worked in the Systems Engineering Laboratory (1968-71) Since 1983 is Professor at the Department of Electronic Engineering of the U.P.C. with teaching and research responsibilities in the area of Digital Systems and VLSI design. His current research include Test and Design for Testability, Defect and Fault Models and Testing and Fault Tolerance methodologies for emerging technologies. 

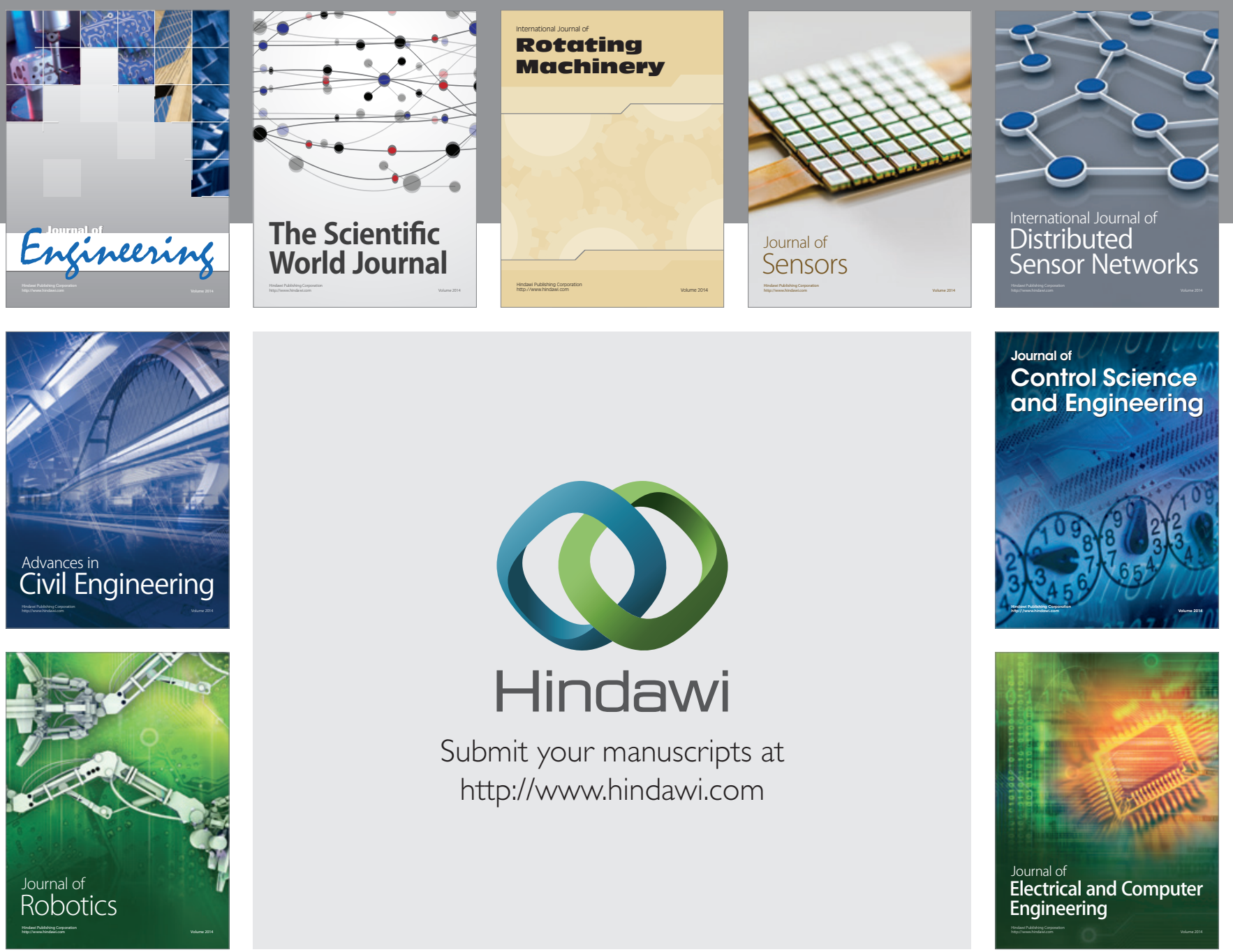

Submit your manuscripts at

http://www.hindawi.com
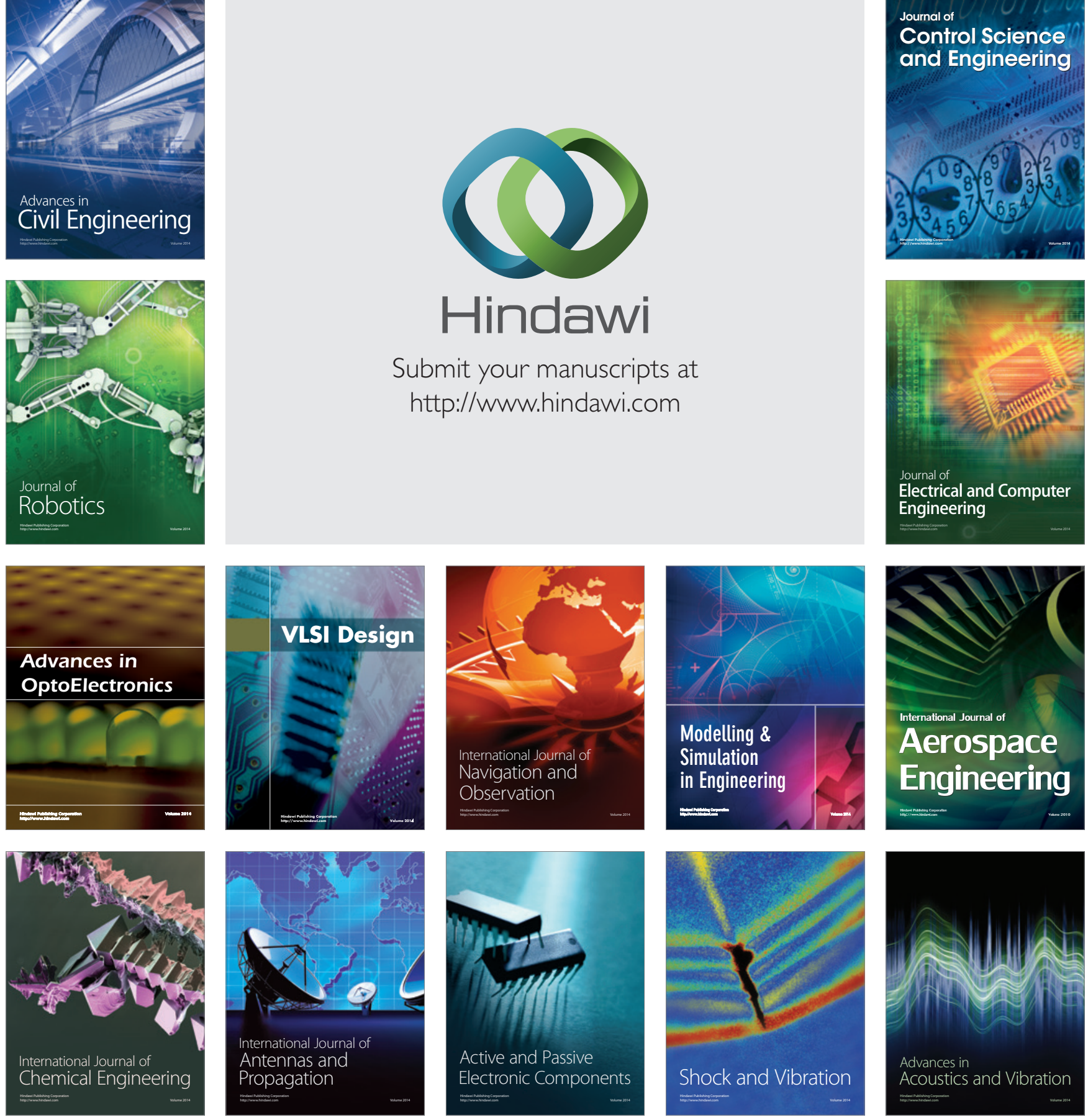